\title{
Description du texte présenté
}

Tome I: MARGVERITES / / DE LA MARGVERITE / / DES PRINCESSES, / / TRESILLVSTRE / / ROYNE / / DE / / NAVARRE. / / [Fleuron.] / / [Vignette: Cupidon, avec devise: PER IPSVM FACTA SVNT OMNIA.] / / A LYON, / / PAR IEAN DE TOVRNES. / / M.D.XLVII. // Auec Priuilege pour six ans. //

B.M., G. I 8 I9o. In-8 ${ }^{\circ}$. Ital. $54^{1}$ pp. chiff. 3-54I, commençant au foa $\mathrm{r}^{0}$; signat. $a-z^{8}, A-L^{8}$ (ts mal sign. ss).

$\mathrm{Au}$ verso de la page de titre: extrait du privilège, daté: Bordeaux «le xxix iour de Mars, Lan mil cinq cens quarantesix auant Pasques ».

pp. 3-1 I $\left(22 \mathrm{r}^{\circ}-26 \mathrm{r}^{\circ}\right)$ : Epître: A trèsillustre et trèschrétienne Princesse, Madame la Princesse de Navarre, I. de la Haye son trèshumble serviteur, souhaite entière prospérité.

p. I $2\left(26 \mathrm{v}^{\circ}\right)$ : Sonnet: Aux Dames des vertus de la trèsillustre et trèsvertueuse Princesse Marguerite de France, Reine de Navarre dévotement affectionées. M. Sc. (c.-à-d., Maurice Scève.) pp. I $^{-1} 4\left(a 7^{0}-v^{0}\right)$; Marguerite de France, par la grâce de Dieu Reine de Navarre, au lecteur. (En vers.)

pp. I $5-70\left(\mathrm{a}^{\circ} \mathrm{r}^{\circ}-\mathrm{e}_{3} \mathrm{v}^{\circ}\right)$ : Le Miroir de l'Ame pécheresse.

pp. $7^{\mathrm{I}-77}\left(\mathrm{e}_{4} \mathrm{r}^{\mathrm{O}}-\mathrm{e} 7^{\mathrm{r}}\right)$ : Discord étant en l'homme par la contrariété de l'Esprit et de la Chair, et paix par vie spirituelle.

pp. 77-1 $35\left(e 7^{0} \mathbf{r}^{0}-14 r^{\circ}\right)$ : Oraison de l'Ame fidèle, à son Seigneur Dieu.

pp. I 35-1 $47\left(14 r^{0}-k 2 r^{\circ}\right)$ : Oraison à notre Seigneur Jésus-Christ. 


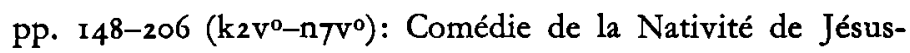
Christ.

pp. 207 [mal numérotée 206 dans les deux exemplaires du B.M.] $-270\left(n 8 r^{0}-r_{7} v^{0}\right)$ : Comédie de l'adoration des Trois Rois à Jésus-Christ.

pp. $271-315\left(\mathrm{r}^{2} \mathrm{r}^{\circ}-\mathrm{v} 6 \mathrm{r}^{\circ}\right)$ : Comédie des Innocents.

pp. $316-380\left(\mathrm{v}^{\circ} \mathrm{v}^{\circ}-\mathrm{A} 6 \mathrm{v}^{\circ}\right)$ : Comédie du Désert.

pp. $38 \mathrm{r}-443\left(\mathrm{~A}_{7^{\mathrm{o}}}{ }^{\mathrm{B}} \mathrm{E} 6 \mathrm{r}^{\circ}\right)$ : Le Triomphe de l'Agneau.

pp. 444-466 (E6vo-Givo): Complainte pour un détenu prisonnier.

pp. ${ }^{6}{ }^{6}-54 \mathrm{I}\left(\mathrm{G}_{2} \mathrm{r}^{\circ}-\mathrm{L}_{7} \mathrm{r}^{\circ}\right)$ : Chansons spirituelles.

p. [542] $\left(L_{7} \mathrm{v}^{\circ}\right)$ : Sonnet: «L'Esprit de Vie en corps de Mort mussé». Au-dessous: "Amour demourra le maistre».

foL81 ${ }^{\circ}$ : marque typographique de De Tournes (Cartier, t.I, p. 36, «senestrochère $I$ »).

foL $8 \mathrm{v}^{\circ}$ : grand ornement en arabesques.

$$
\text { * * }
$$

Tome II (les deux livres sont reliés en un seul volume): SVYTE DES / / MARGVERITES / / DE LA MARGVERITE / / DES PRINCESSES, / / TRESILLVSTRE / / ROYNE / / DE / / NAVARRE. / / [Fleuron.] / / [Vignette: Cupidon avec devise: PER IPSUM FACTA SVNT OMNIA.] / / A LYON, / / PAR IEAN DE TOVRNEs. / / M.D.XLVII. // Auec Priuilege pour six ans. //

In-80. Ital. $34^{2}$ pp. chiff. $2-34^{2}$, commençant au foavo et finissant à y $3 \mathrm{v}^{0}$; signat. $a-\mathrm{x}^{8}, \mathrm{y}^{4}(\mathrm{~d} s, \mathrm{~h} 4, \mathrm{y}$, non sign.).

p. $2\left(\mathrm{av}^{0}\right)$ Sonnet: A trèsillustre et trèsvertueuse Princesse Madame Jeanne Infante de Navarre. M.Sc. (c.-à.d., Maurice Scève.) 
pp. 3-37 (a2r $\left.{ }^{\circ}-c_{3} r^{\circ}\right):$ L'Histoire des Satyres et Nymphes de Diane.

pp. 38-45 $\left(\mathrm{c}_{3} \mathrm{v}^{0}-\mathrm{c} 7 \mathrm{r}^{0}\right)$ : Epitre de la Reine de Navarre, au Roi François son frère.

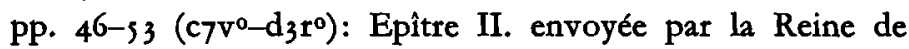
Navarre, avec un David au Roi François, son frère, pour ses étrennes.

pp. $\int 4-57\left(d_{3} v^{\circ}-d_{5} r^{\circ}\right):$ Réponse envoyée par le Roi François à ladite Dame, avec une sainte Catherine pour ses étrennes.

pp. s $8-64\left(\mathrm{~d} 5 \mathrm{v}^{\circ}-\mathrm{d} 8 \mathrm{v}^{0}\right)$ : Epître III. de la Reine de Navarre, au Roi François, son frère.

pp. 65 -7I (eir $\left.{ }^{\circ}-4^{\circ} r^{\circ}\right):$ Epître de la Reine au Roi François son frère.

pp. $7{ }^{2}-75\left(e 4 v^{0}-e 6 r^{\circ}\right)$ : Epitre de la Reine de Navarre, au Roi de Navarre, malade.

pp. $7^{6-1} 77\left(e 6 v^{0}-m^{\circ} r^{\circ}\right)$ : Les quatre Dames et les quatre gentilshommes.

pp. I 78-2 I I $\left(\mathrm{miv}^{\circ}-\mathrm{o}_{2} \mathrm{r}^{\circ}\right)$ : Comédie. Deux Filles, Deux Mariées, La Vieille, Le Vieillard, et les Quatre Hommes.

Pp. $2 \mathrm{I}_{2-264}\left(\mathrm{O}^{2} \mathrm{v}^{0}-\mathrm{r}_{4} \mathrm{v}^{\circ}\right)$ : Farce, de Trop. Prou. Peu. Moins.

pp. $26 s-323\left(r \mathrm{sv}^{\circ}-\mathrm{x}^{2} \mathrm{r}^{\circ}\right)$ : La Coche.

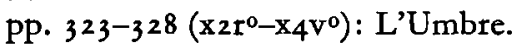

pp. 328-33 I $\left(\mathrm{x}_{4} \mathrm{v}^{0}-\mathrm{x} 6 \mathrm{r}^{\circ}\right)$ : La Mort et Résurrection d'Amour.

pp. $332-336\left(x_{6} \mathrm{v}^{\circ}-\mathrm{x}^{\circ} \mathrm{v}^{0}\right)$ : Chanson faite à une Dame, sur laquelle la Reine a fait la réponse suivante.

pp. 337-34 $\left(\right.$ yir $\left.^{\circ}-\mathrm{y}_{3} \mathrm{r}^{\circ}\right)$ : Les Adieux des Dames de chez la Reine de Navarre, allant en Gascogne, à ma Dame la Princesse de Navarre.

p. $342\left(y 3 v^{0}\right)$ : deux «Enigmes».

foy $4 \mathrm{r}^{\mathrm{r}}$ : blanc.

fo $y 4 v^{\circ}$ : grand ornement en arabesques, cf. le tome I, foL8vo. 
Des exemplaires de cette édition se trouvent à: Lyon, Genève, Oxford, Manchester, Cambridge, Londres (British Museum et Victoria and Albert Museum), Harvard, et dans la collection Terrebasse. 